\title{
Téoros
}

Revue de recherche en tourisme

\section{Les croisières : la quintessence de l'industrie touristique}

\section{Georges Jodoin}

Volume 6, numéro 1, mars 1987

Tourisme et transports

URI : https://id.erudit.org/iderudit/1080524ar

DOI : https://doi.org/10.7202/1080524ar

Aller au sommaire du numéro

Éditeur(s)

Université du Québec à Montréal

ISSN

0712-8657 (imprimé)

1923-2705 (numérique)

Découvrir la revue

Citer cet article

Jodoin, G. (1987). Les croisières : la quintessence de l'industrie touristique.

Téoros, 6(1), 30-32. https://doi.org/10.7202/1080524ar d'utilisation que vous pouvez consulter en ligne.

https://apropos.erudit.org/fr/usagers/politique-dutilisation/ 


\section{Les croisières: la quintessence de I'industrie touristique}

par Georges Jodoin *

A la fois "resort" flottant, entreprise d'animation, grand restaurateur et transporteur de luxe capable de visiter grandes villes et espaces naturels inviolés sur des circuits pratiquement illimités, la croisière constitue sans doute, par la complexité et la diversité de ses opérations, la fine fleur du secteur touristique.

\section{Bref historique}

La période initiale de l'histoire de cette industrie, dominée par les croisières transatlantiques et qui s'étend grosso modo de 1900 à 1960 , date où la concurrence implacable de l'aviation commerciale provoqua la quasi disparition des derniers "dinosaures" de l'océan, a ćté le témoin d'une des plus feroces compétitions commerciales que le monde ait connue. C'était en effet a qui, de l'Angleterre, de la France, de l'Allemagne, de l'Italie ou des États-Unis lancerait le navire le plus imposant, le plus luxueux et surtout le plus rapide entre les deux continents, avec en guise de récompense, le célèbre Blue Riband, trophée symbolique de la suprématie en mer.

On assista ainsi, du Deutschland des Allemands qui remporta le trophée en 1901 en effectuant la traversée en six jours exactement, au SS United States, qui établit un record inégalé en trois jours et 11 heures en 1952, en passant par les Normandie, Queen Mary et bien d'autres à une sorte de méga-course de vitesse sur I'AtlantiqueNord. Celle-ci réclama d'ailleurs son tribut: le Titanic, plus rapide que le Deutchland et d"un luxe sans rival sombra en 1912 , indirectement victime de cette compétition. Le Queen Elizabeth II de la Cunard Line, un des derniers représentants de l'espèce, incarne encore aujourd"hui les débuts de cette époque révolue qui commence, il est intéressant de le rappeler, avec un certain Samuel Cunard, illustre armateur canadien dont le nom est à l'industrie maritime ce qu'Einstein est à la physique ${ }^{(1)}$.

Les années d'après-guerre furent marquées par la concurrence du transport aérien. Jus-

\footnotetext{
"Géographe au ministère du Touriame du Québec, Direction générale du developpement, Analyse et déve. loppement.
}

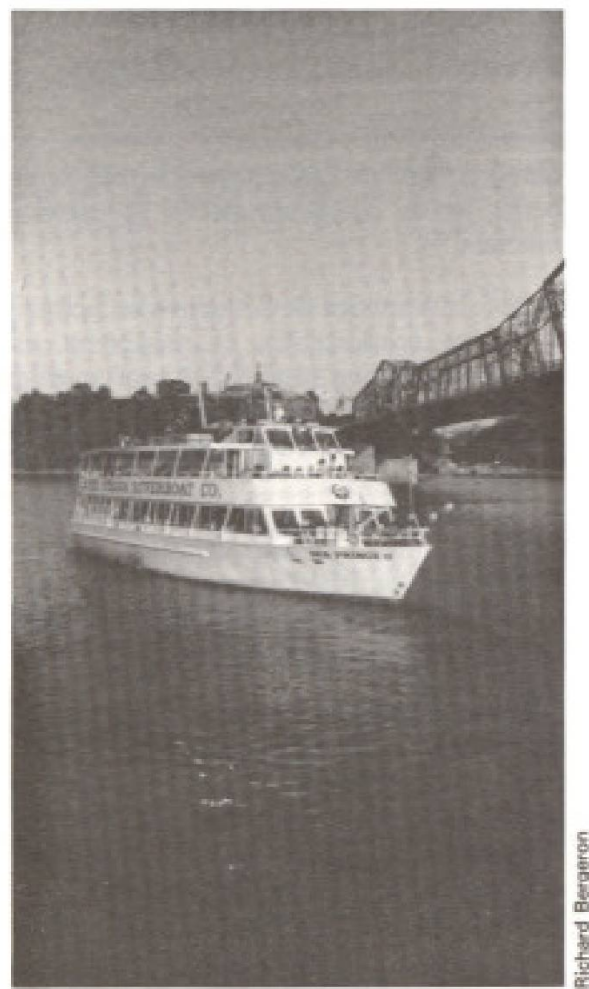

que dans les années ' 60 , il ếtait plus économique de franchir l'Atlantique par bateau que par avion. 1958 fut la demiere année où le bateau traversa plus de passagers que l'avion.

Les compagnies maritimes cherchèrent alors désespérément de nouveaux débouchés à leur flotte - en se tournant graduellement vers la croisière dans les Caraibes notamment - mais plusieurs ne survécurent pas à la transition. Celles qui y parvinent créerent, avec de nouvelles concurrentes dans le domaine, une toute nouvelle industrie. Le tournant des années "70 marque la construction de nombreux bateaux spécifiquement conçus pour une nouvelle clientèle touristique et mieux adaptés aux petits ports d'escale des Caraïbes. Par rapport aux navires de la première genération, les Queen Mary $(81,000$ tonnes $/ 2,000$ passagers) ou SS France ( 66,000 tonnes/2,200 passagers) par exemple, le navire-type des années 70 jauge en général 20,000 tonnes et accommode en moyenne 600 passagers ${ }^{(2)}$.

\section{L'essor de l'industrie}

Le marche des croisières est actuellement en pleine expansion. Entre 1970 et 1986 , la clientèle nord-américaine est passée de 500,000 passagers à $2,500,000$ et l'industrie prévoit un taux de croissance annuel de $10 \%$ au cours des cinq prochaines années.

Les Caraibes accaparent environ $70 \%$ de cette clientèle. Les autres destinations de prédilection des Nord-Américains sont la Riviera mexicaine et l'Alaska. Cette dernière destination a connu un boum remarquable au cours des dernières années. Pas moins de vingt et un navires de croisières se sont rendus en Alaska en 1986 , contre seulement treize en 1982.

Avec 345,000 passagers, la Méditerranée constitue la seconde destination en importance dans le monde. Par aileurs, le nombre de passagers en partance de Miami, le centre névralgique et sans conteste la capitale mondiale de l'industrie, est passé de 400,000 en 1969 , à plus de $1,300,000$ en 1985.

\section{Structure du secteur}

L'industrie des croisières est dominée par une cinquantaine de compagnies maritimes. Les petites compagnies ne possèdent qu'un ou deux navires de croisières. Considérant qu'un nouveau navire de 500 cabines peut coûter $\$ 100$ millions, il faut comprendre qu'il s'agit tout de même d'entreprises d'une certaine envergure.

Les entreprises moyennes operrent trois ou quatre navires et les plus importantes, comme la Norwegian Caribbean Lines, la Costa Lines, la Cunard Line et la Carnival Cruise rarement plus de six, opérant sur une base annuelle.

On peut avancer que l'industrie mondiale est constituće de quelque deux cents navires dont environ cent sont basés dans moins d'une dizaine de ports américains. L'information statistique sur l'industrie est difficile à obtenir. La plupart des données disponibles proviennent de la Cruise Lines International Association (CLIA), une organisation regroupant 27 compagnies maritimes operant 85 navires et offrant plus de 56,000 lits. Bien que l'industrie soit con- 
centrée aux Êtats-Unis, les propriétaires et opérateurs de navires de croisières sont principalement européens: norvégiens, grecs, italiens, anglais, suédois, allemands...

\section{Évolution de l'offre}

On comptait en 1980 un peu plus de 44,000 lits sur le marché nord-américain; en 1984, ce nombre était porté à 53,000 et on estime en 1987 que la capacité atteint 70,000 lits.

Au cours des quatre prochaines années, les compagnies máritimes dépenseront $\$ 1$ milliard pour l'achat de sept nouveaux navires. Le plus remarquable sera sans doute le Sovereign of the Seas de la Cribbean Cruises Lines, qui sera lancé du chantier naval français de St-Nazaire(3) en 1988 . Ce navire de 70,000 tonnes accommodant 2,673 passagers coûtera $\$ 183,5 \mathrm{M}$ (US). La Sitmar Cruise a commandé à ce même chantier deux navires d'une capacité de plus de 1,400 passagers au coût de $\$ 306 \mathrm{M}$. Le Queen Elizabeth II sera par ailleurs complètement rénové au coût de $\$ 120 \mathrm{M}$. La construction de ce navire coủterait aujourd hui $\$ 350 \mathrm{M}$. On constate donc que les grands navires, caractéristique des années ' 30 , réapparaissent sur le marché.

\section{Le marché potentiel}

L'industrie des croisières est le segment de l'industrie touristique connaissant le taux de croissance le plus élevé. Le prix relativement bas du produit et le fait que cette option ne retienne actuellement que $1 \%$ du marché total des vacances ne sont pas étrangers à cette situation. La guerre des prix que se livrent les compagnies maritimes se comparent à celle qui fait rage dans le transport aérien depuis la déréglementation aux États-Unis ${ }^{(4)}$. Dix-neuf Nord-Américains sur vingt n'ont jamais fait de croisière. $40 \%$ des passagers en sont à leurs première expérience. Le taux de satisfaction est très élevé et une vaste majorité désire renouveler l'experience.

Environ le tiers des adeptes de la croisière ont entre 18 et 34 ans, un autre tiers entre 35 et 54 ans et afin le dernier, 55 ans et plus. La CLIA estime que la moitié des passagers pour les Caraibes ont moins de 45 ans et gagnent moins de $\$ 25,000$ par annee. L'industrie vise un marché potentiel de quelques 22 millions d'Américains. Il ne s'agit donc plus d'un marché limité aux personnes âgées et aux revenus élevés.

\section{Les intermédiaires}

Les réservations de croisières augmentent de façon notoire et comptent maintenant pour $14 \%$ de tous les revenus des agences de voyages, A titre de comparaison, les réservations hôtelières comptent pour $10 \%$, les locations de voitures pour $7 \%$ et les voyages en train pour $3 \%$. Ces ventes de croisjères ont connu une hausse de $\$ 2,4$ milliards entre 1983 et 1985 , selon une enquête menée auprès des agences américaines $^{(5)}$.

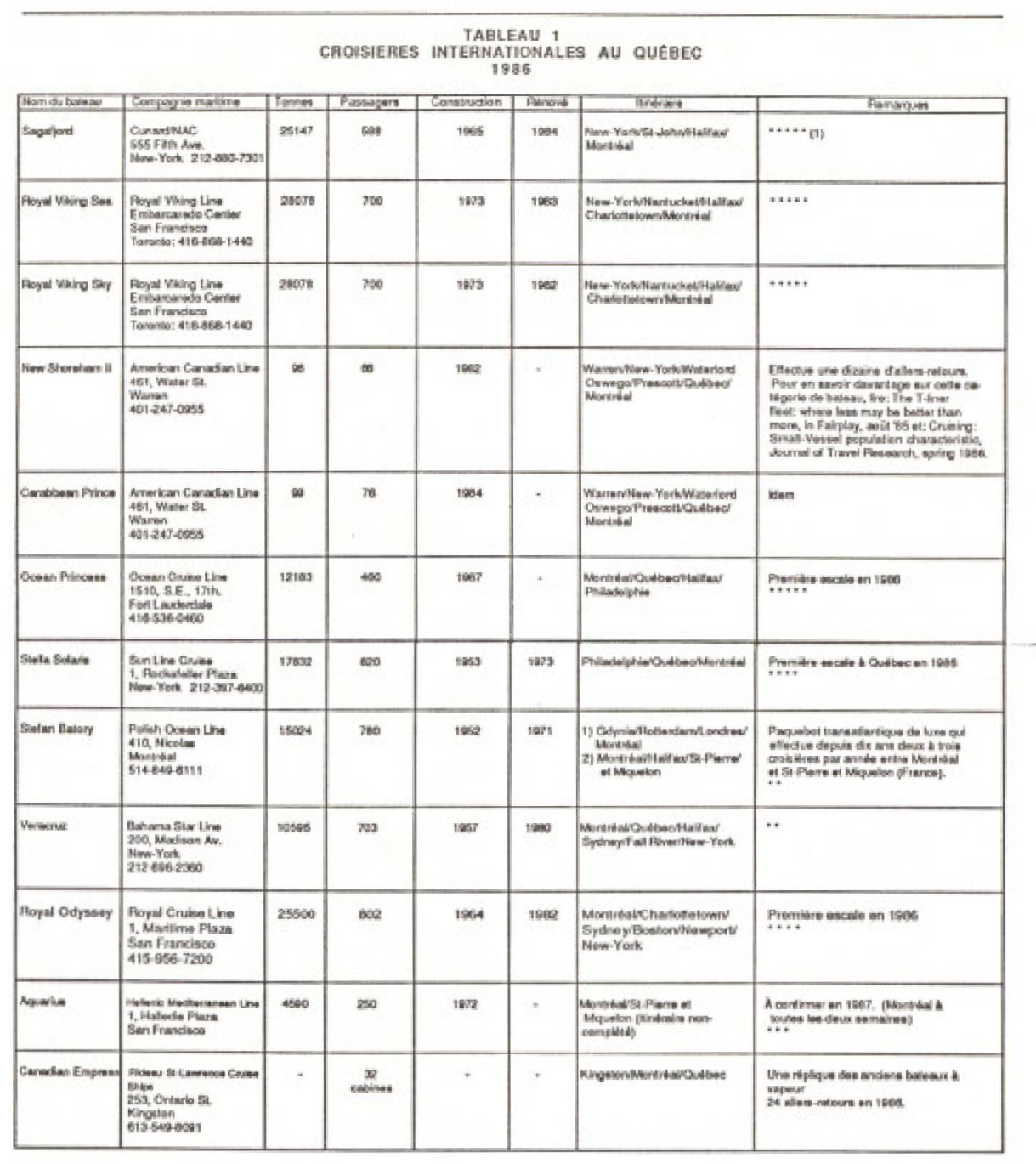

La croissance de l'industrie des croisières est étroitement reliée à la déréglementation dans le transport aérien à la fin des années '70 aux États-Unis et à l'apparition de forfaits air/mer faisant en sorte que l'industrie peut désormais rejoindre des marchés géographiques autrefois hors de portée.

\section{Le Québec: \\ une zone de destination potentielle?}

Trois nouveaux noms se sont ajoutés à la liste des paquebots de luxe qui ont jeté l'ancre au port de Québec au cours de la saison estivale 1986, soit l'Ocean Princess, le Royal Odyssey et le Stella Solaris. Il y a eu ainsi une soixantaine d'escales de navires de croisières à Québec permettant à quelques 20,000 passagers de visiter la ville. À Montréal, la situation est à peu près semblable, le nombre de passagers étant de l'ordre de 22,000 à 24,000 par année. Le tableau 1 résume la situation à partir d'informations diverses.

Le Québec peut-il espérer accroître sa part de marché à titre de zone de destination potentielle dans le contexte nord-américain?
Un examen rapide de certains facteurs positifs portent à le croire.

\section{La surcapacité mondiale}

Depuis 1979, la capacité de la flotte des navires de croisières a augmenté plus rapidement que la demande et cette capacité est appelée à augmenter de $35 \%$ au cours des quatre prochaines années. Cette surcapacité force les compagnies maritimes à modifier et diversifier leurs zones de destinations traditionnelles - la destination Caraibes commence à être saturée - au profit de zones plus secondaires comme le Québec.

\section{Le potentiel de croissance de la demande}

L'offre excédentaire a également pour conséquence de créer une véritable guerre des prix, de sorte que ce type de vacances est devenu de plus en plus abordable pour le touriste moyen. En fait, l'option croisière se compare désormais, en terme de coût, au séjour dans un "resort" ou encore au traditionnel circuit touristique en automobile. La popularité croissante de cette option favorise les destinations en mesure d'offrir un produit interressant, y compris le Québec. 


\section{Taux de change et terrorisme}

Selon l'American Association of Port Authorities, chaque passager dépense $\$ 255$ par jour lors d'une escale dont la durée moyenne est de 1,5 jour. Dans cette perspective, le taux de change des pays-hótes joue un rôle important et représente pour le Québec un facteur positif additionnel.

La Mediterranée représente par ailleurs une zone de destination concurentielle au système St-Laurent-Grands-Lacs sur le marchế américain des croisiêres $(75,000$ passagers). Or, les incidents survenus sur l'Achille Lauro en Méditerranée ont eu des répercussions négatives considérables, non pas sur le produit croisière lui-même, mais sur cette destination particulière ${ }^{(6)}$. L'Alaska et, dans une moindre mesure, le Québec ont profité de la vague d'annulations en Mediterranée.

\section{Proximité du marché}

Les Américains représentent de loin la principale clientèle des navires de croisières dans le monde (plus de 2,000,000 de passagers) $^{(7)}$. Le Sud-Est (les Caraibes) accapare $70 \%$ de ce marché. Le Sud-Ouest (la Riviera mexicaine), le Nord-Ouest (Alaska: 120,000 passagers en 1984 ) et le Nord-Est (Nouvelle-Angleterre-Canada) se partagent le reste. Le fait que l'industrie soit concentrée sur la côte Est (1,3 millions de passagers partent du port de Miami) représente certes un avantage pour le Québec.

\section{Les forfaits air-mer}

L'itinéraire Nord-Est - St-Laurent correspond en quelque sorte a un trajet vers l'interieur d'un entonnoir. Les perspectives d'un retour par la meme route paraissent peu intéressantes. Les forfaits air-mer, une pratique devenue maintenant courante, contribuent à éliminer ce désavantage propre à notre destination.

Contrairement aux trois autres destinations concurrentes susmentionnées, le Québec constitue une région emettrice de touristes, un avantage que les compagnies maritimes ne sauraient ignorer.

\section{Deux bassins de clientèle}

Les ports du Québec ont l'avantage de pouvoir bénéficier directement à la fois d'un marché de quelque 50 millions $^{(3)}$ de touristes américains via l'embouchure du StLaurent et d'un autre marché de 22,5 millions de touristes via la voie maritime du St-Laurent.

\section{La rénovation des ports de Montréal et de Québec}

Une récente enquête aupres de 26 ports canadiens $^{(9)}$ révèle que le taux de croissance des passagers de croisière est de $13,7 \%$ en 1987 et qu'il augmentera en moyenne de $16,7 \%$ au cours des dix prochaines années. Par ailleurs, $38 \%$ des ports ont procédé à une étude d'impact écono- mique des croisières. L'impact moyen a été évalué à $\$ 962$ par passager.

Les deux tiers de ces ports ont investi dans la mise en place d'infrastructures destinces aux passagers depuis les cinq dernieres années. L'investissement a été en moyenne de $\$ 11,2$ millions. Les importantes améliorations apportées récemment aux infrastructures portuaires de Québec et de Montréal sont de nature à améliorer sensiblement la position des ports d'escale au Quebec.

\section{Une combinaison de produits}

Les croisieres sont en quelque sorte des voyages touristique (tourisme de circuit) à l'intérieur d'un centre de villégiature, les bateaux modernes étant de véritables "resorts". Les croisières peuvent également offrir le produit "plein-air" et le produit "grandes villes". Selon une étude du marché américain des voyages d'agrément ${ }^{(19)}$, le Québec possède certains points forts en particulier sur le produit "grandes villes" et le produit circuit.

Québec et Montréal sont d'ailleurs au nombre des produits canadiens les plus populaires auprès du marché americain.

\section{Perspectives}

La fréquentation occasionnelle ou régulière de navires de croisières dans les eaux du St-Laurent n'est pas le resultat d'un travail concerté en vue d'amener les compagnies maritimes et les grossistes à considérer cette destination. Actuellement, les efforts consacrés en ce sens sont très limités, pour ne pas dire nuls. Considérant les retombées actuelles, qui peuvent être estimés à quelques millions de dollars, les énergies a investir ne sauraient être que restreintes. II y a cependant lieu de penser qu'un minimum d'efforts pourrait étre fait, preférablement en collaboration avec les provinces maritimes (Halifax, Charlettetown) et la Nouvelle-Angleterre (Boston).

Le gouvernement de la Colombie Britanique a entrepris un certain nombre d'action conjointes avec l'industrie des croisières ${ }^{(1)}$. Les autorites portuaires de Boston et l'Office de tourisme de Boston ont tenu un séminaire à bord du Royal Viking Sky daris le but d'établir une stratégie pour accroitre les retombes de cette industrie en pleine expansion ${ }^{(12)}$. Le Halifax Board of Trade a fait préparer une étude des retombées de l'industrie des croisières sur son économie locale ${ }^{(13)}$. L'étude conclut qu'un effort de marketing doit être entrepris aupres des compagnies. À la Societé de développement économique du StLaurent (SODES), on estime inacceptable et incompréhensible qu'aucun effort n'ait jamais été entrepris dans ce sens.

La réalisation d'un vidéo mettant en valeur les nombreux attraits le long de l'itinéraire

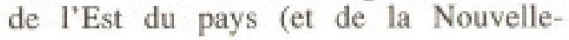

Angleterre éventuellement) constituerait sans doute un instrument de promotion fort utile lors d'Eventuelles demarches auprès des décideurs... $f$

\section{Notes}

11) Le dictionnaire bibliographique du Canada con sacre quelque 15 pagas a Sir Samuel Cunard, ne a Halifas il $\mathrm{y}$ a cent ans.

12) A noter que le Rapport tonnage/pasagger cons. titue un bon indice de la densto d'occupation et par consequent du confort du bateau.

(3) II s'agit du chantier de la compagnie Alsthom Atlantique, le même qui avait construit le SS France (maintenant le Norway) et le Normandie.

(4) Le tarif Montróal-Paris en 1965, qui etait de $\$ 347$, est de $\$ 348$ en 1985 , soit $\$ 90.48$ en dollars de 1965.

(5) Voir Travel Weekly"s Louis Harris Survey, juin 1966.

(6) L'Achille Laure $[23,629$ tonnes), qui a dete le thet tre d'une prise d'otages avant entraind la mort d'un ressortissant américain, a été retiné de la Mediterranée orientale. Par ailleurs, rOcean Islander de I'Ocean Cruise britanique qui operait habituellement entre Venise et Nice avec une clientäle A $B 0 \%$ amáricaine assure maintenant des croisiares dans les eaux scandinaves à la place de l'Ocean Princess transferé bu secteur américain A partir de Philadelphie vers Québec et Montréal [voir tableat 1).

(7) En comparaison, les trois principaux marchés sulvants, I'Allemagne de I'Ouest (Mediterrande), l'Angleterne (Méditerranés) et I'Australia (la Coste) recueillent respectivement $150,000,105,000$ et 100,000 passagers en 1985 .

(8) Soit la Nouvelle-Angleterre 17,7 millions de touristes), le Centre-Atlantique $(22,3 \mathrm{M}\}$ et Atlantique-Sud $(22,3 \mathrm{M})$.

(9) "The emerging domestic cruise industry" Phillips Cartner \& CO., sept. 1985.

(10) Le marchế américain des woyages d'agrément Tourisme Canada, 1985.

(11) Voir "Canadian propose new links with cruise lines". Travel Weekly, 31 juin 1986.

(12) Voir" "Boston hopes to double its cruse market by $1990^{\circ}$, Travel Weakly, 5 jarvier 1987.

(13) Cruise shipes in the port of Halifax, par Criterion Regearch Ass., novembre 1994. 